\title{
Comparison of salt experiments and empirical time of concentration equations
}

Asghar Azizian PhD

Assistant Professor, Department of Water Engineering, Faculty of

Engineering and Technology, Imam Khomeini International University,

Qazvin, Iran (azizian@eng.ikiu.ac.ir) (Orcid:0000-0002-6556-2931)

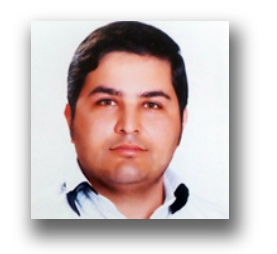

The time of concentration $\left(T_{c}\right)$ is one of the most contributing parameters for assessing the response of a catchment to rainfall events and estimating the peak flood in many hydrological models. The aim of this work was to evaluate 36 time of concentration equations based on $T_{c}$ values obtained based on the salt dilution tracing approach. In addition, hierarchical cluster analysis (CA) was applied to identify the degree of similarity between all the $T_{c}$ formulas and categorise them into several groups. The findings, based on seven sub-watersheds of the Meime river basin in Iran, demonstrate that the Picking, Pickering, DNOS, California and Kirpich-Ten equations provide reliable estimation of $T_{c}$; the average bias of these formulas was found to be in the range 2.6-15 min while, for all 36 equations, the bias was 2.6-132.8 min. Furthermore, based on CA, all $T_{c}$ equations were categorised into four overall groups with the maximum similarity within the members of each group.

\section{Notation}

A catchment area $\left(\mathrm{km}^{2}\right)$

$A_{\text {imp }} \quad$ fraction of impervious area (\%)

$C$ runoff coefficient

CN SCS curve number

$D \quad$ equivalent diameter of catchment $(\mathrm{km})$

$H \quad$ quota difference between the ends of the main channel or longest flow path $(\mathrm{m})$

$H_{\mathrm{m}} \quad$ mean altitude of catchment (m)

I percentage of impervious areas (\%)

$i_{2 y} \quad$ two-year rainfall intensity $(\mathrm{mm} / \mathrm{h})$

$K \quad$ type of surface coefficient in SCS Formula

$k \quad$ type of surface coefficient in DNOS Formula

$L_{\mathrm{b}} \quad$ length of watershed along the main channel from the hydraulically most distant point to the outlet $(\mathrm{km})$

$L_{\mathrm{c}} \quad$ length of main channel $(\mathrm{km})$

$L_{\text {ca }} \quad$ mean length starting from concentration spot along the length up to the spot where $L_{\mathrm{c}}$ is perpendicular to the centroid of the catchment $(\mathrm{km})$

$L_{\mathrm{LF}} \quad$ length of longest flow path for each sub-basin $(\mathrm{km})$

$L_{\mathrm{m}} \quad$ length of all streams in watershed

$L_{\mathrm{t}} \quad$ length of all streams larger than $30 \mathrm{inch}$ in width

$N \quad$ retardance coefficient

$n \quad$ Manning's roughness coefficient

$p \quad$ relation between vegetation cover and total area of basin
$S_{\mathrm{b}} \quad$ average slope of watershed $(\mathrm{m} / \mathrm{m})$

$S_{\mathrm{c}} \quad$ average slope of main channel or longest flow path $(\mathrm{m} / \mathrm{m})$

$S_{\text {scs }} \quad$ maximum capacity of retention $(\mathrm{mm})$

$T_{\mathrm{c}} \quad$ time of concentration (h)

$\Delta H_{\text {channel }}$ quota difference between ends of main channel (m)

$\Delta H_{\text {basin }} \quad$ mean elevation of catchment (m)

$\Phi \quad$ conductance or channelisation factor

\section{Introduction}

The time of concentration $\left(T_{\mathrm{c}}\right)$ is a concept used in hydrology to measure the response of a watershed to a rain event (Haan et al., 1994). It is defined as the time required for all contributing parts of the catchment to producing runoff (or discharge) at the watershed's outlet (Fang et al., 2008), and is one of the most important parameters for evaluating the response of a catchment (McCuen et al., 1984; Wong, 2009) and estimating peak floods in hydrologic designs. For instance, the geomorphological instantaneous unit hydrograph (GIUH) and kinematic waved (KW)-GIUH models that are used for estimation of peak flood require an accurate estimation of $T_{\mathrm{c}}$ (Azizian and Shokoohi, 2015a, 2015b; Huang et al., 2015). In addition, the Clark unit hydrograph method requires an estimation of $T_{\mathrm{c}}$ in order to calculate the time-area histogram, the time to peak and the peak flood (Dhakal et al., 2014: Sadeghi et al., 2015). Appropriate estimations of $T_{\mathrm{c}}$ can minimise errors in the prediction of design discharges and, 
subsequently, flood damage (Bondelid et al., 1982; de Almeida et al., 2014). If the values for $T_{\mathrm{c}}$ are underestimated, they will lead to overestimated values for results related to peak discharge and vice versa (Fang et al., 2008). However, the concept of $T_{\mathrm{c}}$ is highly questioned because there are difficulties in its determination and because it is influenced by the rainfall intensity and the initial moisture conditions of the catchment. There is therefore significant uncertainty about identifying the best $T_{\mathrm{c}}$ method and what best defines its concept (Grison et al., 2008). Because of the great importance of $T_{\mathrm{c}}$, there is a huge number of empirical/semi-empirical and analytical $T_{\mathrm{c}}$ equations and each has resulted from studies that have been performed in specific regions. For this reason, researchers and engineers are often confused by the many equations and select a formula without evaluating and comparing its accuracy with other methods (Grimaldi et al., 2012; McCuen et al., 1984; Wong and Asce, 2005).

Several studies have been carried out to address the importance of $T_{\mathrm{c}}$ in hydrological modelling, identify the accuracy of the $T_{\mathrm{c}}$ equations and introduce new approaches for estimating $T_{\mathrm{c}}$. For example, McCuen et al. (1984), Wong and Asce (2005), Fang et al. (2008), Sharifi and Hosseini (2011) and Liang and Melching (2012) reviewed, compared and evaluated different empirical/semi-empirical and analytical $T_{\mathrm{c}}$ equations. Moreover, some researchers have focused on developing new approaches for the estimation of $T_{\mathrm{c}}$ or modifying empirical and analytical equations such as the kinematic wave and Morgali-Linsley equations (Baiamonte and Singh, 2015; De Almeida et al., 2016; El-Hames, 2013; Grimaldi and Petroselli, 2015; Manoj et al., 2013; Ogbonna, 2004). There are many approaches for estimating a catchment's $T_{\mathrm{c}}$, but the major ones are as follows.

(a) Estimating the time span between the end (or centre) of effective rainfall and the inflection point of the hydrograph falling limb (McCuen, 2009; Singh, 1988).

(b) Using empirical equations that depend on geomorphological parameters (McCuen et al., 1984; Singh, 1988) and rainfall attributes (in some cases). The equations that fall into this category are mainly based on regression analysis and have been developed only for specific catchments (Fang et al., 2007).

(c) Injecting salt dilution or tracer soluble in the upstream of a river and measuring the travel time in the river's downstream (Dingman, 2002).

Results based on either of these approaches will not always be unique and each has some benefits and disadvantages. In other words, a global exact method for estimating $T_{\mathrm{c}}$ is not available and hence the true value of this parameter can never be determined (McCuen et al., 1984). Therefore, for a specific region, one can concentrate on selecting the most accurate formula(s) and modifications if necessary (Sharifi and Hosseini, 2011). In contrast to many research studies, in this work, the third approach was adopted to estimate $T_{\mathrm{c}}$, which was then used as a reference value for an assessment of empirical/semi-empirical $T_{\mathrm{c}}$ equations. Specifically, the aim of this work was to

- evaluate 36 empirical/semi-empirical $T_{\mathrm{c}}$ equations by using the salt injection approach

- classify all $36 T_{\mathrm{c}}$ equations by using hierarchical cluster analysis (CA) to distinguish and categorise the most similar equations.

\section{Method}

\section{$2.1 \quad$ Study area}

The study area, the Meime river basin (MRB), is part of Dehloran province in Iran and its elevation ranges from 1115 to $2244 \mathrm{~m}$ above mean sea level. The total area and perimeter of the MRB are about $83.92 \mathrm{~km}^{2}$ and $47.6 \mathrm{~km}$, respectively. The MRB lies between the coordinates $33^{\circ} 12^{\prime} 34^{\prime \prime} \mathrm{N}$ and $33^{\circ} 19^{\prime}$ $03^{\prime \prime} \mathrm{N}$ latitude and $46^{\circ} 49^{\prime} 44^{\prime \prime} \mathrm{E}$ and $46^{\circ} 58^{\prime} 26^{\prime \prime} \mathrm{E}$ longitude. The climate is semi-arid (or steppe climate) and the annual rainfall varies from 170 to $648 \mathrm{~mm}$ with an average annual rainfall of $291 \mathrm{~mm}$. The daily mean temperature of this watershed varies in the range $12.3-38.7^{\circ} \mathrm{C}$ and the mean relative humidity varies from $18 \%$ (August and July) to a maximum of $66 \%$ (January). To extract the required geomorphological parameters for all the $T_{\mathrm{c}}$ equations, a 1:25000 scale topographic map was used. This map was geo-referenced by Mercator's (UTM, time zone 38) transverse projection within QGIS (previously known as Quantum GIS) (a cross-platform free and open-source desktop geographic information system (GIS) application), and then a digital elevation model (DEM) with $50 \mathrm{~m}$ resolution was obtained. The location of the MRB and its DEM with a resolution of $50 \mathrm{~m}$ is shown in Figure 1.

\subsection{Approach for obtaining the reference value of $T_{\mathrm{c}}$}

The methodology used to obtain the 'reference' $T_{\mathrm{c}}$ values was based on measurement of the travel time of a salt tracer. The salt dilution method is a simple and robust method for flow measurement and estimation of $T_{\mathrm{c}}$, which does not require a hydrologist to enter the deepest sections of a river. This method has been used for flow measurements in several applications and other studies (e.g. Church and Kellerhals, 1970; Hudson and Fraser, 2002; Spence and McPhie, 1997). The basis of the method is the conservation of mass of a tracer substance before and after dilution by a flowing stream. The concentration of the tracer measured downstream after it has become uniformly distributed across the channel is related to the discharge of the stream - the lower the concentration the greater the discharge. Table salt (sodium chloride $(\mathrm{NaCl})$ ) is commonly used as the tracer and its concentration is measurable with high sensitivity (Drost, 1989; Kumar and Nachiappan, 2000; Moore, 2004; Tazoil, 2011). When using sodium chloride as the tracer, electrical conductivity (EC) is 


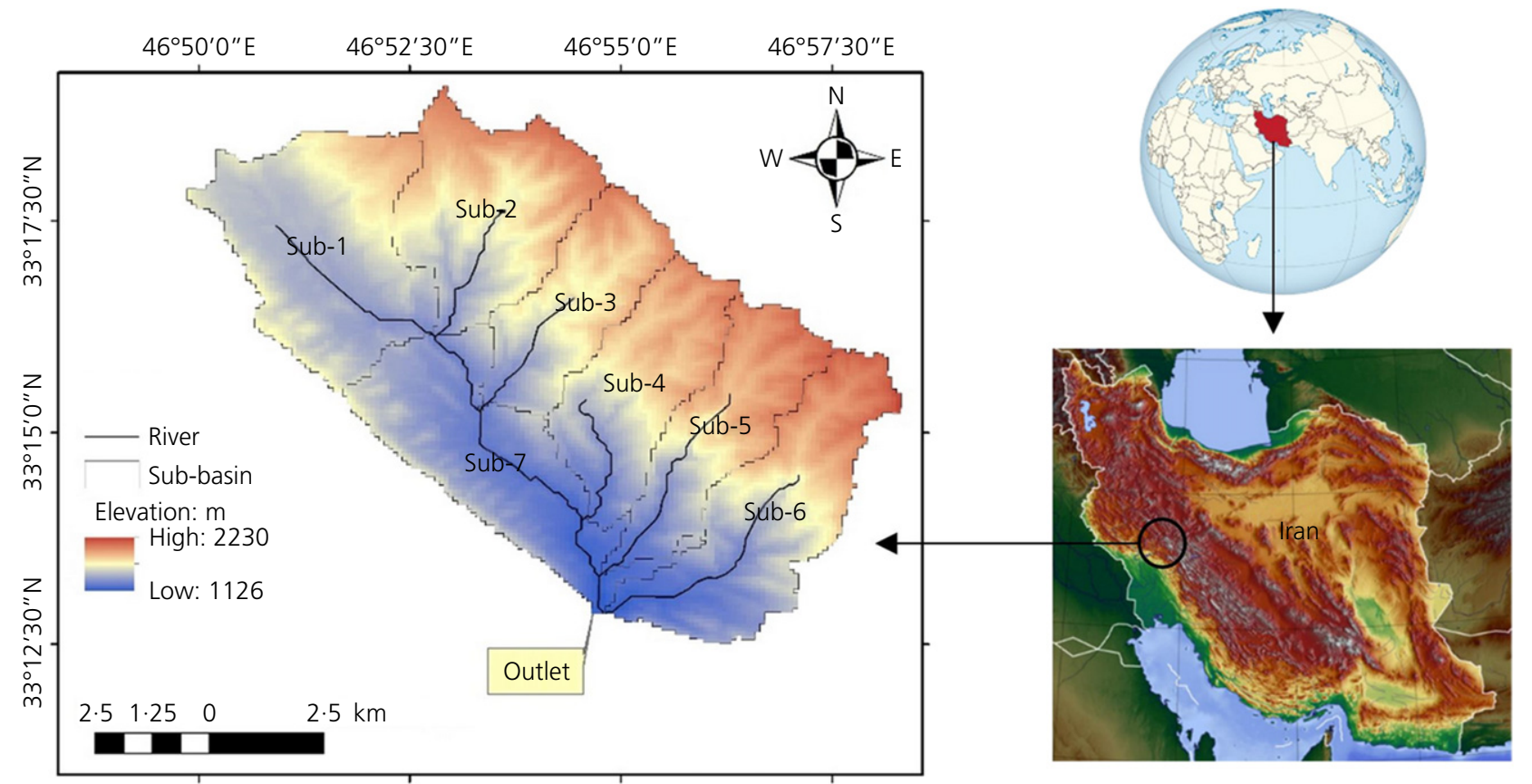

Figure 1. Location and DEM of the study area (map data () Google, AutoNavi, Basarsoft, Mapa GISrael, ORION-ME; imagery @ Landsat, Data SIO, NOAA, US Navy, GEBCO)

substituted for mass concentration. The time between the entry of sodium chloride in the upstream and the time of the peak EC in the downstream is the travel time of flow (or $T_{\mathrm{c}}$ ) in that segment. The summation of travel times in the consecutive segments defines the catchment's $T_{\mathrm{c}}$ and in this study (see Figure 2) is considered as the 'reference' $T_{\mathrm{c}}$ value. The tracer measurements were carried out over $3 \mathrm{~d}$ without any rainfall. Measuring tracer values on a rainy day imposes several difficulties, such as measuring the peak EC accurately and the entry of other pollutions or mudflows from overland parts or tributaries, which would affect the dosage of tracer in the water. In this research, the sodium chloride dilution was made to enter the upstream of each stream suddenly and the variation of EC over time was measured by means of a chronometer in the downstream.

After measuring the travel time for each segment, the geomorphological parameters used in the selected equations needed to be extracted. In this study, the D8 flow tracing algorithm (O'Callaghan and Mark, 1984) in the QGIS environs was used to extract the streams' network and geomorphological features. The geomorphological parameters of the seven sub-basins of the Meime watershed are summarised in Tables 1 and 2.

\subsection{Empirical/semi-empirical $T_{\mathrm{c}}$ equations}

All $T_{\mathrm{c}}$ equations are based on four types of input - slope, catchment size, flow resistance and rainfall attributes. These characteristics are either for the overland flow parts of the watershed, the pipe system or the channel flow system (McCuen et al., 1984). Therefore, $T_{\mathrm{c}}$ formulas can be categorised on the basis of their input requirements and the dominant flow regime. Because the channels and drainage network in the MRB are well-developed, channel flows are predominant and overland flows play a minor role in runoff production. For example, in sub-3, two points (with the greatest distance to the stream network) were selected to measure the travel time of overland flows and the results showed that the maximum $T_{\mathrm{c}}$ value was about $3.3 \mathrm{~min}$. This value of overland $T_{\mathrm{c}}$ in sub-3 relative to the longest flow path's $T_{\mathrm{c}}(42.5 \mathrm{~min})$ was small (less than $8 \%$ ) and could thus be ignored.

Equations for estimating $T_{\mathrm{c}}$ that include channel features should be used when channel flow makes a significant contribution to the total travel time of runoff (McCuen et al., 1984). Thus, in order to compare the empirical equations and the sodium chloride tracer method used in this study, only $T_{\mathrm{c}}$ formulas that fall into the channel flow regime were selected. As shown in Table 3, $T_{\mathrm{c}}$ equations depend on many coefficients, variables and physiographic features. The values of these coefficients and a summary of the watershed characteristics are given in Table 4.

\subsection{Methodology of evaluating and identifying the best $T_{\mathrm{c}}$ equations}

Identifying the best $T_{\mathrm{c}}$ formulas needs reasonable goodness-offit criteria. There is a huge number of criteria when it comes to 


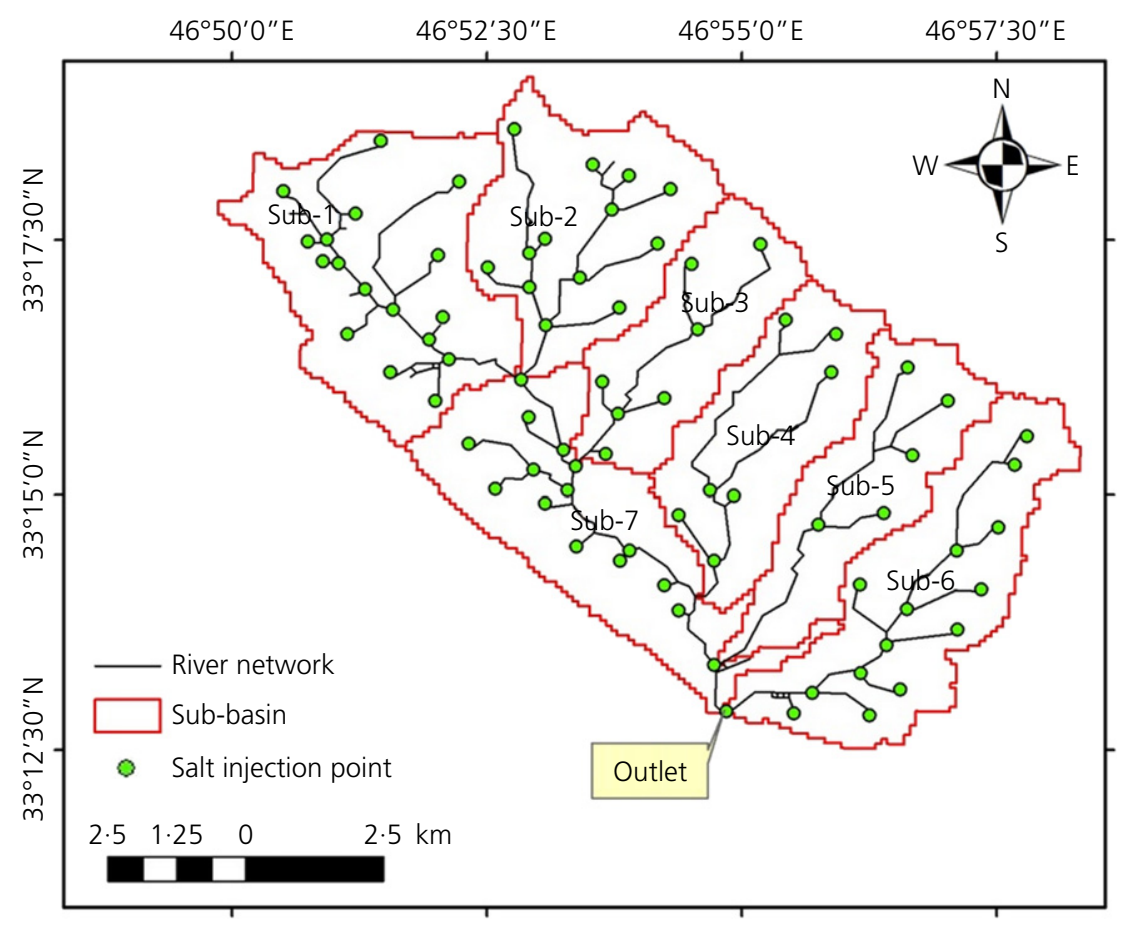

Figure 2. Locations of salt injection points in each river segment

comparing observed and estimated values and each of them gives different results. Similar to the study of Sharifi and Hosseini (2011), a ranking-based selection procedure was developed in this research. The mean error (ME), mean relative error (MRE) and root mean square error (RMSE) were selected as assessment criteria. The difference in the means of the estimated and reference $T_{\mathrm{c}}$ values can be used as a measure of the actual bias; a positive value of the ME indicates overprediction while a negative value illustrates that the estimated value is less than the reference $T_{\mathrm{c}}$. The ME for an equation can be dominated by errors in large $T_{\mathrm{c}}$ values (McCuen et al., 1984). Thus, standardised bias statistics (SBS or MRE) were used to indicate the bias in each $T_{\mathrm{c}}$ formula. All the $T_{\mathrm{c}}$ equations were ranked based on each criterion and these were then summed up to give the overall ranking. These indices were obtained using

1. $\quad \mathrm{RMSE}=\sqrt{\sum_{i=1}^{N_{\mathrm{b}}} \frac{\left(T_{\mathrm{c}_{\mathrm{est}}}-T_{\mathrm{c}_{\mathrm{obs}}}\right)^{2}}{N_{\mathrm{b}}}}$

2. $\mathrm{ME}=\operatorname{Bias}=\frac{1}{N_{\mathrm{b}}} \sum_{i=1}^{N_{\mathrm{b}}}\left(T_{\mathrm{cest}}-T_{\mathrm{c}_{\mathrm{obs}}}\right)$
3. $\mathrm{MRE}=\mathrm{SBS}=\frac{1}{N_{\mathrm{b}}} \sum_{i=1}^{N_{\mathrm{b}}} \frac{\left(T_{\mathrm{c}_{\mathrm{est}}}-T_{\mathrm{c}_{\mathrm{obs}}}\right)}{T_{\mathrm{c}_{\mathrm{obs}}}}$

in which $N_{\mathrm{b}}$ is the number of sub-basins, $T_{\text {cest }}$ is the estimated $T_{\mathrm{c}}$ value based on empirical equations and $T_{\mathrm{c}_{\mathrm{obs}}}$ is the reference $T_{\mathrm{c}}$ value.

\subsection{Classification of all $T_{\mathrm{c}}$ equations using CA}

CA (or clustering) is the task of grouping a set of objects in such a way that objects in the same group (called a cluster) are more similar and hold common features. CA is one of a class of techniques that classifies cases into groups that are relatively homogeneous within themselves and relatively heterogeneous between each other (Lowrimore and Manton, 2016). This analysis is commonly used in many research fields such as geomorphology (Melchiorre et al., 2008), environmental engineering (Hatvani et al., 2011) and water resources (Li et al., 2012; Yoo et al., 2011). There are two main methods - hierarchical and non-hierarchical CA - and, for simplicity, the hierarchical method was used in this study. Hierarchical CA requires the researcher to establish a prior number of clusters in the final solution (Ratrout, 2010). If there is uncertainty about the total number of clusters in the dataset, the analysis must be re-run for each possible solution. In this situation, hierarchical clustering is preferred as it inherently allows one to compare the clustering result with an increasing number of clusters; no 
Table 1. Measured $T_{\mathrm{c}}$ values and geomorphological parameters of each sub-basin

\begin{tabular}{|c|c|c|c|c|c|c|c|}
\hline Sub-basin & $\begin{array}{c}\text { Reach } \\
\text { number }\end{array}$ & $\Delta H_{\text {channel: }}: m$ & $\begin{array}{l}\text { Horizontal } \\
\text { distance: } m\end{array}$ & $\begin{array}{c}\text { Velocity: } \\
\text { m/s }\end{array}$ & $\begin{array}{l}\text { Channel } \\
\text { length: } \mathrm{km}\end{array}$ & $\begin{array}{l}\text { Channel } \\
\text { slope: \% }\end{array}$ & $\begin{array}{l}\text { Measured } T_{\mathrm{c}} \text { : } \\
\min \end{array}$ \\
\hline \multirow[t]{8}{*}{ Sub-1 } & 1 & 26 & 730 & $2 \cdot 0$ & $1 \cdot 21$ & $3 \cdot 56$ & $9 \cdot 60$ \\
\hline & 2 & 40 & 1350 & $2 \cdot 1$ & $2 \cdot 24$ & 3.96 & $16 \cdot 20$ \\
\hline & 3 & 40 & 1130 & $2 \cdot 6$ & 1.88 & $3 \cdot 54$ & $13 \cdot 20$ \\
\hline & 4 & 40 & 1760 & $2 \cdot 4$ & 2.92 & $2 \cdot 27$ & $22 \cdot 20$ \\
\hline & 5 & 40 & 700 & $2 \cdot 2$ & $1 \cdot 16$ & $5 \cdot 71$ & $7 \cdot 20$ \\
\hline & 6 & 40 & 700 & $2 \cdot 1$ & $1 \cdot 17$ & $5 \cdot 71$ & $7 \cdot 20$ \\
\hline & 7 & 80 & 400 & $2 \cdot 0$ & 0.68 & $20 \cdot 00$ & $3 \cdot 50$ \\
\hline & Total & & & & $11 \cdot 26$ & & $79 \cdot 10$ \\
\hline \multirow[t]{11}{*}{ Sub-2 } & 1 & 26 & 670 & $2 \cdot 3$ & 0.67 & $3 \cdot 88$ & $5 \cdot 17$ \\
\hline & 2 & 40 & 540 & $2 \cdot 7$ & 0.59 & $7 \cdot 41$ & $3 \cdot 34$ \\
\hline & 3 & 60 & 720 & $3 \cdot 2$ & 0.72 & $8 \cdot 33$ & 3.95 \\
\hline & 4 & 100 & 780 & $3 \cdot 1$ & 0.79 & $12 \cdot 82$ & 3.65 \\
\hline & 5 & 60 & 680 & $2 \cdot 5$ & 0.68 & 8.82 & 3.95 \\
\hline & 6 & 80 & 770 & $3 \cdot 0$ & 0.77 & $10 \cdot 39$ & 3.95 \\
\hline & 7 & 100 & 600 & $3 \cdot 1$ & 0.67 & $16 \cdot 67$ & $2 \cdot 74$ \\
\hline & 8 & 200 & 610 & $4 \cdot 1$ & 0.64 & $32 \cdot 79$ & $2 \cdot 13$ \\
\hline & 9 & 140 & 390 & $5 \cdot 1$ & 0.65 & $35 \cdot 90$ & $1 \cdot 52$ \\
\hline & Total & & & & $6 \cdot 18$ & & $30 \cdot 40$ \\
\hline & 1 & 42 & 830 & $2 \cdot 6$ & 0.83 & $5 \cdot 60$ & $5 \cdot 62$ \\
\hline \multirow[t]{10}{*}{ Sub-3 } & 2 & 40 & 710 & $2 \cdot 2$ & 0.71 & 5.63 & 4.94 \\
\hline & 3 & 80 & 780 & $3 \cdot 1$ & 0.78 & $10 \cdot 26$ & $4 \cdot 28$ \\
\hline & 4 & 80 & 700 & $3 \cdot 2$ & 0.71 & 11.43 & 3.95 \\
\hline & 5 & 100 & 860 & $3 \cdot 1$ & 0.87 & 11.63 & $4 \cdot 61$ \\
\hline & 6 & 80 & 870 & $3 \cdot 2$ & 0.88 & $9 \cdot 20$ & 4.94 \\
\hline & 7 & 80 & 710 & $3 \cdot 3$ & 0.71 & $11 \cdot 27$ & 3.95 \\
\hline & 8 & 60 & 780 & $2 \cdot 4$ & 0.78 & $7 \cdot 69$ & 4.94 \\
\hline & 9 & 140 & 760 & $3 \cdot 3$ & 0.77 & $18 \cdot 42$ & 3.62 \\
\hline & 10 & 120 & 350 & 5.0 & 0.59 & 34.29 & 1.65 \\
\hline & Total & & & & 7.63 & & $42 \cdot 50$ \\
\hline \multirow[t]{11}{*}{ Sub-4 } & 1 & 44 & 800 & $2 \cdot 6$ & 0.83 & $5 \cdot 50$ & $5 \cdot 24$ \\
\hline & 2 & 60 & 850 & $2 \cdot 9$ & 0.85 & 7.06 & 4.93 \\
\hline & 3 & 80 & 940 & $3 \cdot 2$ & 0.94 & 8.51 & 4.93 \\
\hline & 4 & 80 & 730 & $3 \cdot 3$ & 0.73 & $10 \cdot 96$ & $3 \cdot 71$ \\
\hline & 5 & 120 & 680 & $3 \cdot 4$ & 0.69 & $17 \cdot 65$ & $3 \cdot 38$ \\
\hline & 6 & 180 & 730 & $4 \cdot 5$ & 0.75 & $24 \cdot 66$ & $2 \cdot 77$ \\
\hline & 7 & 40 & 640 & $2 \cdot 5$ & 0.65 & $6 \cdot 25$ & $4 \cdot 31$ \\
\hline & 8 & 40 & 710 & $2 \cdot 6$ & 0.73 & 5.63 & $4 \cdot 62$ \\
\hline & 9 & 80 & 790 & $3 \cdot 0$ & 0.79 & $10 \cdot 13$ & $4 \cdot 32$ \\
\hline & 10 & 180 & 840 & $4 \cdot 2$ & 0.86 & 21.43 & $3 \cdot 39$ \\
\hline & Total & & & & $7 \cdot 82$ & & $41 \cdot 60$ \\
\hline \multirow[t]{13}{*}{ Sub-5 } & 1 & 41 & 650 & $2 \cdot 5$ & 0.43 & $6 \cdot 31$ & $2 \cdot 84$ \\
\hline & 2 & 40 & 570 & $2 \cdot 6$ & 0.38 & 7.02 & $2 \cdot 43$ \\
\hline & 3 & 40 & 780 & $2 \cdot 5$ & 0.52 & $5 \cdot 13$ & $3 \cdot 45$ \\
\hline & 4 & 40 & 700 & $2 \cdot 5$ & 0.46 & $5 \cdot 71$ & 3.04 \\
\hline & 5 & 80 & 630 & $3 \cdot 5$ & 0.43 & $12 \cdot 70$ & 2.03 \\
\hline & 6 & 120 & 610 & $3 \cdot 8$ & 0.41 & 19.67 & 1.82 \\
\hline & 7 & 140 & 650 & $4 \cdot 2$ & 0.44 & $21 \cdot 54$ & 1.74 \\
\hline & 8 & 140 & 640 & 4.2 & 0.43 & 21.88 & 1.72 \\
\hline & 9 & 80 & 850 & $3 \cdot 1$ & 0.57 & $9 \cdot 41$ & 3.04 \\
\hline & 10 & 80 & 940 & $3 \cdot 2$ & 0.63 & 8.51 & 3.25 \\
\hline & 11 & 80 & 500 & 3.5 & 0.34 & $16 \cdot 00$ & 1.62 \\
\hline & 12 & 140 & 420 & $4 \cdot 0$ & 0.29 & $33 \cdot 33$ & $1 \cdot 22$ \\
\hline & Total & & & & $5 \cdot 33$ & & $28 \cdot 20$ \\
\hline
\end{tabular}


Table 1. Continued

\begin{tabular}{|c|c|c|c|c|c|c|c|}
\hline Sub-basin & $\begin{array}{l}\text { Reach } \\
\text { number }\end{array}$ & $\Delta H_{\text {channel: }}: m$ & $\begin{array}{l}\text { Horizontal } \\
\text { distance: } m\end{array}$ & $\begin{array}{l}\text { Velocity: } \\
\mathrm{m} / \mathrm{s}\end{array}$ & $\begin{array}{l}\text { Channel } \\
\text { length: km }\end{array}$ & $\begin{array}{l}\text { Channel } \\
\text { slope: \% }\end{array}$ & $\begin{array}{l}\text { Measured } T_{\mathrm{c}} \text { : } \\
\min \end{array}$ \\
\hline \multirow[t]{14}{*}{ Sub-6 } & 1 & 22 & 630 & $2 \cdot 2$ & 0.51 & $3 \cdot 49$ & 3.80 \\
\hline & 2 & 40 & 930 & $2 \cdot 7$ & 0.75 & $4 \cdot 30$ & 4.68 \\
\hline & 3 & 40 & 750 & $2 \cdot 8$ & 0.61 & $5 \cdot 33$ & 3.57 \\
\hline & 4 & 40 & 690 & $2 \cdot 8$ & 0.56 & $5 \cdot 80$ & $3 \cdot 34$ \\
\hline & 5 & 40 & 690 & $2 \cdot 8$ & 0.56 & $5 \cdot 80$ & $3 \cdot 34$ \\
\hline & 6 & 100 & 660 & $4 \cdot 0$ & 0.54 & $15 \cdot 15$ & 2.23 \\
\hline & 7 & 80 & 710 & $3 \cdot 6$ & 0.58 & $11 \cdot 27$ & $2 \cdot 67$ \\
\hline & 8 & 100 & 700 & $3 \cdot 9$ & 0.57 & $14 \cdot 29$ & $2 \cdot 45$ \\
\hline & 9 & 200 & 760 & $4 \cdot 7$ & 0.64 & $26 \cdot 32$ & $2 \cdot 27$ \\
\hline & 10 & 80 & 780 & $3 \cdot 6$ & 0.63 & $10 \cdot 26$ & $2 \cdot 90$ \\
\hline & 11 & 60 & 650 & $3 \cdot 3$ & 0.53 & $9 \cdot 23$ & $2 \cdot 68$ \\
\hline & 12 & 80 & 730 & $3 \cdot 7$ & 0.59 & $10 \cdot 96$ & $2 \cdot 67$ \\
\hline & 13 & 160 & 720 & $4 \cdot 5$ & 0.60 & $22 \cdot 22$ & $2 \cdot 20$ \\
\hline & Total & & & & $7 \cdot 67$ & & $38 \cdot 80$ \\
\hline \multirow[t]{18}{*}{ Sub-7 } & 1 & 5 & 600 & 1.4 & 0.41 & 0.83 & $4 \cdot 80$ \\
\hline & 2 & 20 & 930 & $2 \cdot 3$ & 0.65 & $2 \cdot 15$ & $4 \cdot 80$ \\
\hline & 3 & 20 & 930 & $2 \cdot 2$ & 0.64 & $2 \cdot 15$ & 4.80 \\
\hline & 4 & 20 & 880 & $2 \cdot 3$ & 0.61 & $2 \cdot 27$ & 4.40 \\
\hline & 5 & 20 & 950 & $2 \cdot 0$ & 0.66 & $2 \cdot 10$ & $5 \cdot 40$ \\
\hline & 6 & 20 & 1070 & $2 \cdot 2$ & 0.74 & 1.87 & $5 \cdot 50$ \\
\hline & 7 & 20 & 870 & $2 \cdot 3$ & $0 \cdot 60$ & $2 \cdot 30$ & 4.40 \\
\hline & 8 & 20 & 750 & $2 \cdot 1$ & 0.52 & $2 \cdot 67$ & $4 \cdot 20$ \\
\hline & 9 & 20 & 1150 & $2 \cdot 2$ & 0.79 & $1 \cdot 74$ & $6 \cdot 00$ \\
\hline & 10 & 20 & 760 & $2 \cdot 2$ & 0.52 & $2 \cdot 63$ & 3.90 \\
\hline & 11 & 40 & 1070 & $2 \cdot 9$ & 0.74 & $3 \cdot 74$ & $4 \cdot 20$ \\
\hline & 12 & 20 & 650 & $2 \cdot 0$ & 0.45 & 3.08 & $3 \cdot 70$ \\
\hline & 13 & 40 & 1130 & $2 \cdot 9$ & 0.78 & $3 \cdot 54$ & 4.50 \\
\hline & 14 & 20 & 1060 & $2 \cdot 3$ & 0.73 & 1.89 & $5 \cdot 40$ \\
\hline & 15 & 40 & 1100 & $2 \cdot 9$ & 0.76 & 3.64 & $4 \cdot 40$ \\
\hline & 16 & 40 & 780 & $3 \cdot 1$ & 0.54 & $5 \cdot 13$ & 2.90 \\
\hline & 17 & 100 & 620 & $2 \cdot 8$ & 0.43 & $16 \cdot 13$ & $2 \cdot 60$ \\
\hline & Total & & & & $10 \cdot 57$ & & $75 \cdot 90$ \\
\hline
\end{tabular}

Table 2. $T_{\mathrm{c}}$ and geomorphological characteristics of the MRB sub-basins

\begin{tabular}{lccccccc} 
Sub-basin & Area: $\mathbf{k m}^{\mathbf{2}}$ & $\begin{array}{c}\text { Main channel } \\
\text { length: } \mathbf{k m}\end{array}$ & $\boldsymbol{\Delta} \boldsymbol{H}_{\text {basin }}: \mathbf{m}$ & $\boldsymbol{\Delta} \boldsymbol{H}_{\text {channel: } \mathbf{m}}$ & Basin slope: $\mathbf{m} \mathbf{m}$ & Channel slope: $\mathbf{m} / \mathbf{m}$ & Observed $\boldsymbol{T}_{\mathbf{c}}: \mathbf{m i n}$ \\
\hline Sub-1 & 16.26 & 11.26 & 681 & 292 & 0.060 & 0.026 & 79.1 \\
Sub-2 & 12.01 & 6.18 & 831 & 806 & 0.134 & 0.130 & 30.4 \\
Sub-3 & 8.82 & 7.63 & 834 & 822 & 0.109 & 0.100 & 42.5 \\
Sub-4 & 10.22 & 7.82 & 907 & 892 & 0.116 & 0.110 & 41.6 \\
Sub-5 & 9.62 & 5.33 & 1029 & 1021 & 0.193 & 0.191 & 28.2 \\
Sub-6 & 13.83 & 7.67 & 1126 & 1032 & 0.146 & 0.134 & 38.8 \\
Sub-7 & 13.16 & 10.57 & 578 & 506 & 0.050 & 0.040 & 75.9
\end{tabular}

decision about the final number of clusters needs to be made beforehand. The first step a researcher must take is to determine the statistic that will be used to calculate the distance or similarity between cases. The most commonly used distance measure is the squared Euclidean distance, given by

4. $d=\sum_{i=1}^{z}\left(a_{i}-b_{i}\right)^{2}$ in which $a_{i}$ and $b_{i}$ refer to the two cases being compared for the variable $i$ and $z$ is the total number of variables included in the analysis (Ratrout, 2010). If the value of $d$ is close to zero, the more similarity exits between two cases.

Finally, to select the optimal number of clusters in the dataset, cluster validity indices should be calculated. There are several validity indices, including the root mean square standard deviation (RMSSTD) of all the variables within each cluster, 
Table 3. Summary of the selected $T_{\mathrm{c}}$ equations

\begin{tabular}{|c|c|c|c|c|}
\hline \multicolumn{2}{|c|}{ Name } & \multirow{2}{*}{$\begin{array}{l}\text { Reference } \\
\text { ADoT (1993) }\end{array}$} & \multirow{2}{*}{$\begin{array}{l}\text { Equation } \\
T_{\mathrm{c}}=0.0097956 A^{0.1}\left(1000 L_{b}\right)^{0.25} L_{c a}^{0.25} S_{b}^{-0.2}\end{array}$} & \multirow{2}{*}{$\begin{array}{l}\text { Comments } \\
\text { Data of agricultural basins }\end{array}$} \\
\hline 1 & Arizona DoT & & & \\
\hline 2 & Basso & $\begin{array}{l}\text { Eslamian and } \\
\text { Mehrabi (2005) }\end{array}$ & $T_{\mathrm{c}}=0.957 \mathrm{~L}_{\mathrm{b}}^{1.155} \mathrm{H}_{\mathrm{m}}^{-0.385}$ & Data not available \\
\hline 3 & $\begin{array}{l}\text { Bransby } \\
\text { Williams }\end{array}$ & Moth (1998) & $T_{c}=0.605 L_{c}\left(100 S_{c}\right)^{-0.2} A^{-0.1}$ & Specially recommended for rural basins \\
\hline 4 & California & $\mathrm{CDH}(1960)$ & $T_{\mathrm{C}}=0.95 \mathrm{LFF}^{1.155} \mathrm{H}^{-0.385}$ & Data of small mountain basins in USA \\
\hline 5 & Carter & Carter (1961) & $T_{\mathrm{c}}=0.0977 L_{\mathrm{b}}^{0.6} \mathrm{~S}_{\mathrm{b}}^{-0.3}$ & $\begin{array}{l}\text { Data of an urban basin in USA }\left(A<20.72 \mathrm{~km}^{2} \text {; }\right. \\
\quad S<0.005)\end{array}$ \\
\hline 6 & Chow et al. & Chow et al. (1988) & $T_{\mathrm{C}}=0.1602 L_{\mathrm{c}}^{0.64} S_{\mathrm{c}}^{-0.32}$ & $\begin{array}{l}\text { Data of } 20 \text { rural basins in USA }\left(0.01-18.5 \mathrm{~km}^{2} \text {; }\right. \\
0.0051<S<0.09)\end{array}$ \\
\hline 7 & $\begin{array}{l}\text { Corps of } \\
\text { Engineers }\end{array}$ & Linsley et al. (1977 & $T_{\mathrm{c}}=0.191 L_{\mathrm{c}}^{0.76} S_{\mathrm{c}}^{-0.19}$ & Data of 25 rural basins in the USA $\left(A \leq 12 \mathrm{~km}^{2}\right)$ \\
\hline 8 & Desbordes & Silveira (2005) & $T_{\mathrm{c}}=0.0869 A^{0.3039} \mathrm{~S}_{\mathrm{b}}^{-0.3832} A_{\mathrm{imp}}^{-0.4523}$ & Data not available \\
\hline 9 & DNOS & Silveira (2005 & $T_{c}=0.419 k^{-1} A^{0.2} L_{c}^{0.2} S_{c}^{-0.4}$ & $\begin{array}{l}\text { Data of six rural basins in USA }\left(A<0.45 \mathrm{~km}^{2} \text {; }\right. \\
0.03<S<0.1)\end{array}$ \\
\hline 10 & Dooge & Dooge (1973) & $T_{\mathrm{C}}=0.365 \mathrm{~A}^{0.41} \mathrm{~S}_{\mathrm{C}}^{-0.17}$ & Data of ten rural basins in Ireland (145-948 $\mathrm{km}^{2}$ ) \\
\hline 11 & Epsey & $\begin{array}{l}\text { Hotchkiss and } \\
\text { McCallum (1995) }\end{array}$ & $T_{\mathrm{c}}=6.89 L_{\mathrm{c}}^{0.36} S_{\mathrm{c}}^{-0.18}$ & Data of 11 rural basins in USA \\
\hline 12 & $\begin{array}{l}\text { Epsey- } \\
\text { Winslow }\end{array}$ & McCuen (1998) & $T_{\mathrm{c}}=0.45 \Phi L_{\mathrm{c}}^{0.29} S_{\mathrm{c}}^{-0.11} J^{-0.6}$ & $\begin{array}{l}\text { Data of } 17 \text { rural and urbanised basins }\left(1-35 \mathrm{mi}^{2}\right. \\
\left.\left(\approx 3-90 \mathrm{~km}^{2}\right)\right)\end{array}$ \\
\hline 13 & Eagleson & Eagleson (1962) & $T_{\mathrm{C}}=0.275 n L_{\mathrm{c}} R^{-0.667} S_{\mathrm{C}}^{-0.5}$ & $\begin{array}{l}\text { Calibrated from data for catchments less than } 8 \mathrm{mi}^{2} \\
\left(\approx 21 \mathrm{~km}^{2}\right)\end{array}$ \\
\hline 14 & FAA & FAA (1970) & $T_{\mathrm{C}}=0.3788(1.1-C) L_{\mathrm{LF}}^{0.5} S_{\mathrm{b}}^{-0.332}$ & Data of airports' drainage \\
\hline 15 & Flavell & Flavell (1983) & $T_{\mathrm{c}}=2 \cdot 31 \mathrm{~A}^{0.54}$ & $\begin{array}{l}\text { Obtained from observed times of hydrograph rise on } \\
\text { basins in the south west of Western Australia }\end{array}$ \\
\hline 16 & Ribeiro & Ribeiro (1961) & $T_{c}=0.267(1.05-0.2 p)^{-1} L_{c} S_{c}^{-0.04}$ & $\begin{array}{l}\text { Data of seven rural basins in USA and a rural basin } \\
\text { in India }\left(A<19000 \mathrm{~km}^{2} ; 0.03<S<0 \cdot 1\right)\end{array}$ \\
\hline 17 & Giandotti & Giandotti (1934) & $T_{\mathrm{c}}=\left(4 \sqrt{A}+1.5 L_{\mathrm{c}}\right) /\left(0.8 \sqrt{H_{\mathrm{m}}}\right)$ & Data of basins in Italy $\left(170-70000 \mathrm{~km}^{2}\right)$ \\
\hline 18 & $\begin{array}{l}\text { Haktanir- } \\
\text { Sezen }\end{array}$ & $\begin{array}{l}\text { Haktanir and Sezen } \\
\text { (1990) }\end{array}$ & $T_{\mathrm{C}}=0.7473 L_{\mathrm{LF}}^{0.841}$ & Data of ten basins in Turkey $\left(11-9867 \mathrm{~km}^{2}\right)$ \\
\hline 19 & $\begin{array}{l}\text { Johnstone- } \\
\text { Cross }\end{array}$ & $\begin{array}{l}\text { Johnstone and Cross } \\
\text { (1949) }\end{array}$ & $T_{\mathrm{c}}=0.4623 \mathrm{LLF}_{\mathrm{LF}}^{0.5} \mathrm{~S}_{\mathrm{b}}^{-0.5}$ & Data of 19 rural basins in USA $\left(64 \cdot 8-4206 \cdot 1 \mathrm{~km}^{2}\right)$ \\
\hline 20 & $\begin{array}{l}\text { Kerby- } \\
\text { Hathaway }\end{array}$ & $\begin{array}{l}\text { Kerby (1959); } \\
\text { Hathaway (1945) }\end{array}$ & $T_{\mathrm{c}}=0.606 N^{0.47} L_{\mathrm{LF}}^{0.47} S_{\mathrm{b}}^{-0.235}$ & $\begin{array}{l}\text { Analysis of overland flow in experimental surfaces } \\
\left(L_{\mathrm{LF}}<0.37 \mathrm{~km}\right)\end{array}$ \\
\hline 21 & Kirpich-Ten & Kirpich (1940) & $T_{\mathrm{c}}=0.0653 L_{\mathrm{LF}}^{0.77} \mathrm{~S}_{\mathrm{b}}^{-0.385}$ & Data of small watersheds in Tennessee and \\
\hline 22 & Kirpich-Pen & Kirpich (1940) & $T_{\mathrm{c}}=0.01104 L_{\mathrm{LF}}^{0.77} S_{\mathrm{b}}^{-0.5}$ & Pennsylvania (0.004-0.453 km \\
\hline 23 & McCuen et al. & $\begin{array}{l}\text { McCuen et al. } \\
\text { (1984) }\end{array}$ & $T_{\mathrm{c}}=2.2535 i_{2 \mathrm{y}}^{-0.7164} L_{\mathrm{LF}}^{0.5552} S_{\mathrm{C}}^{-0.207}$ & $\begin{array}{l}\text { Starting from data of } 48 \text { urban basins in USA } \\
\left(0.4-16 \mathrm{~km}^{2} ; 0.0007<S<0.03\right)\end{array}$ \\
\hline 24 & Pasini & Pasini (1914) & $T_{c}=0.108 A^{0.332} L_{c}^{0.332} S_{c}^{-0.5}$ & Data of rural basins in Italy \\
\hline 25 & Pickering & $\begin{array}{l}\text { Mata-Lima et al. } \\
\text { (2007) }\end{array}$ & $T_{\mathrm{c}}=0.9482 L_{\mathrm{c}}^{1.155} \mathrm{H}^{-0.385}$ & Equivalent to Kirpich equation \\
\hline 26 & Picking & Silveira (2005) & $T_{c}=0.0883 L_{c}^{0.667} S_{c}^{-0.332}$ & Data of rural basins \\
\hline 27 & $\begin{array}{l}\text { Pilgrim- } \\
\text { McDermott }\end{array}$ & $\begin{array}{l}\text { Pilgrim and } \\
\text { McDermott } \\
\text { (1982) }\end{array}$ & $T_{\mathrm{C}}=0.76 \mathrm{~A}^{0.38}$ & $\begin{array}{l}\text { Developed from } 96 \text { basins in eastern New South } \\
\text { Wales }\end{array}$ \\
\hline 28 & SCS-lag & Mockus (1961) & $T_{\mathrm{c}}=0.057[(1000 / \mathrm{CN})-9]^{0.7} L_{\mathrm{LF}}^{0.8} S_{\mathrm{b}}^{-0.5}$ & Data of 24 rural basins in USA $\left(A<8 \mathrm{~km}^{2}\right)$ \\
\hline 29 & $\begin{array}{l}\text { SCS-ave } \\
\text { velocity }\end{array}$ & NRCS $(1972,1986)$ & $T_{c}=0.278 \sum L_{c} / K S_{c}^{0.5}$ & $\begin{array}{l}\text { Method relates watershed slope and surface to flow } \\
\text { velocity }\end{array}$ \\
\hline 30 & $\begin{array}{l}\text { Simas- } \\
\text { Hawkins }\end{array}$ & $\begin{array}{l}\text { Simas and Hawkins } \\
\text { (2002) }\end{array}$ & $T_{c}=0.322 A^{0.594} L_{c}^{-0.594} S^{-0.15} S_{\text {scs }}^{0.312}$ & Data of 168 basins in USA $\left(0.001-14 \mathrm{~km}^{2}\right)$ \\
\hline 31 & Schaake et al. & $\begin{array}{l}\text { Schaake et al. } \\
\text { (1967) }\end{array}$ & $T_{c}=0.0828 L_{c}^{0.24} S^{-0.16} A_{i m p}-0.26$ & Data not available \\
\hline 32 & Sheridan & Sheridan (1994) & $T_{\mathrm{c}}=2 \cdot 20 L_{\mathrm{LF}}^{0.92}$ & $\begin{array}{l}\text { Nine flatland watersheds located in Georgia and } \\
\text { Florida, USA }\left(2 \cdot 62-334.34 \mathrm{~km}^{2}\right)\end{array}$ \\
\hline
\end{tabular}


Table 3. Continued

\begin{tabular}{|c|c|c|c|c|}
\hline \multicolumn{2}{|c|}{ Name } & \multirow{2}{*}{$\begin{array}{l}\text { Reference } \\
\text { Temez (1978) }\end{array}$} & \multirow{2}{*}{$\begin{array}{l}\text { Equation } \\
T_{\mathrm{C}}=0.3 L_{\mathrm{c}}^{0.76} S_{\mathrm{C}}^{-0.19}\end{array}$} & \multirow{2}{*}{$\begin{array}{l}\text { Comments } \\
\text { Data of natural basins in Spain }\end{array}$} \\
\hline 33 & Temez & & & \\
\hline 34 & Van Sickle & Van Sickle (1962) & $T_{\mathrm{c}}=0.0081 L_{\mathrm{t}}^{0.13} L_{\mathrm{m}}^{0.13} \mathrm{~S}^{-0.065}$ & $\begin{array}{l}\text { Calibrated from data collected in Houston, USA, } \\
\text { with drainage areas less than } 36 \mathrm{mi}^{2}\left(\approx 93 \mathrm{~km}^{2}\right)\end{array}$ \\
\hline 35 & Ventura & $\begin{array}{l}\text { Mata-Lima et al. } \\
\text { (2007) }\end{array}$ & $T_{\mathrm{c}}=4 A^{0.5} L_{\mathrm{C}}^{0.5} H^{-0.5}$ & Data of rural basins in Italy \\
\hline 36 & Williams & Williams (1922) & $T_{\mathrm{c}}=0.272 \mathrm{~A}^{0.4} L_{\mathrm{b}} D^{-1} \mathrm{~S}_{\mathrm{b}}{ }^{-0.2}$ & Data of basins in India $\left(A<129.5 \mathrm{~km}^{2}\right)$ \\
\hline
\end{tabular}

Table 4. Summary of adopted values of the coefficients and watershed characteristics

\begin{tabular}{|c|c|c|c|c|c|}
\hline \multirow[b]{2}{*}{ Watershed characteristics } & \multirow[b]{2}{*}{ Average } & \multirow[b]{2}{*}{ Standard deviation } & \multicolumn{2}{|c|}{ Range } & \multirow[b]{2}{*}{ CoV } \\
\hline & & & Minimum & Maximum & \\
\hline Area: $\mathrm{km}^{2}$ & $12 \cdot 00$ & $2 \cdot 63$ & 8.82 & $16 \cdot 26$ & 0.22 \\
\hline Longest flow path: km & $10 \cdot 06$ & $3 \cdot 54$ & $7 \cdot 43$ & $14 \cdot 35$ & $0 \cdot 23$ \\
\hline Basin slope: \% & $11 \cdot 54$ & $4 \cdot 90$ & $5 \cdot 00$ & $19 \cdot 30$ & 0.43 \\
\hline Main channel length: km & $8 \cdot 07$ & $2 \cdot 16$ & $5 \cdot 33$ & $11 \cdot 26$ & $0 \cdot 27$ \\
\hline Main channel slope: \% & $10 \cdot 44$ & $5 \cdot 70$ & $2 \cdot 60$ & $19 \cdot 10$ & 0.54 \\
\hline Coefficient $\Phi$ (Epsey-Winslow) & $0 \cdot 24$ & 0.13 & 0.14 & 0.36 & 0.53 \\
\hline SCS curve number & $73 \cdot 00$ & $6 \cdot 20$ & $67 \cdot 00$ & $78 \cdot 00$ & 0.05 \\
\hline Two-year rainfall intensity: $\mathrm{mm} / \mathrm{h}^{\mathrm{a}}$ & 8.62 & 1.65 & $6 \cdot 50$ & $9 \cdot 50$ & $0 \cdot 19$ \\
\hline Coefficient k (DNOS) & $3 \cdot 25$ & $1 \cdot 13$ & $2 \cdot 56$ & $4 \cdot 85$ & 0.35 \\
\hline Coefficient C (FAA) & 0.36 & $0 \cdot 11$ & 0.25 & 0.42 & 0.30 \\
\hline Coefficient N (Kerby-Hathaway) & 0.20 & 0.09 & $0 \cdot 15$ & 0.31 & 0.45 \\
\hline Manning's coefficient & $0 \cdot 016$ & 0.01 & 0.01 & 0.029 & 0.63 \\
\hline Percentage of impervious area: \% & $7 \cdot 00$ & $2 \cdot 71$ & $4 \cdot 20$ & $9 \cdot 30$ & 0.39 \\
\hline Coefficient $p$ (Ribeiro) & 0.34 & $0 \cdot 14$ & 0.23 & 0.37 & 0.42 \\
\hline
\end{tabular}

${ }^{a}$ According to variations of temporal rainfall pattern due to climate change in the study area, the rate of rainfall intensity varies between $6 \cdot 50$ and $9 \cdot 50 \mathrm{~mm} / \mathrm{h}$

Dunn's index, Davies-Bouldin's index and the pseudo-F index (Milligan and Cooper, 1985). In this study, the RMSSTD index was used for validation of hierarchical CA. This index determined by calculating the within-group sum of squares of each cluster and normalising it by the product of the number of elements in the cluster and the number of variables (Sharma, 1995). The RMSSTD index is given by

\section{5. $\operatorname{RMSSTD}=\sqrt{W_{k} /\left[v\left(N_{z}-1\right)\right]}$}

where $W_{k}$ is the within-group sum of squares of cluster $k, N_{z}$ is the number of elements in cluster $z$ and $v$ is the number of variables (the variable used in this study was the $T_{\mathrm{c}}$ value obtained based on 36 equations). This index calculates at each step of the hierarchical algorithm a measure of homogeneity of the clusters. In each step, the RMSSTD index is compared with the previous value and if the difference between two steps is lower than $5 \%$, this will be the optimum number of clusters.

\section{Results and discussion}

\subsection{Identifying the best $T_{\mathrm{c}}$ equations}

The equations listed in Table 3 were used to estimate $T_{\mathrm{c}}$ for all the sub-basins of the MRB. The ME and MRE values are summarised in Table 5. The results indicate that the $T_{\mathrm{c}}$ formulas showed significantly different values of $\mathrm{ME}$ (or bias). Five of the equations - the Picking, Pickering, DNOS, California and Kirpich-Ten equations - provided relatively small biases, had the highest overall rankings and gave accurate $T_{\mathrm{c}}$ estimations. The maximum values of the MRE and ME for these equations were $14.4 \%$ and $6.05 \mathrm{~min}$, respectively, and it can be concluded that these equations are the most appropriate for estimating $T_{\mathrm{c}}$ in the Meime watershed. The development conditions (restrictions) of the DNOS, Kirpich-Ten and Pickering equations were very similar to each other. As summarised in Table 3, the DNOS, Kirpich-Ten and Pickering methods were developed for small or rural basins of the USA. The flow path slopes and catchment areas used in the development of these formulas were $0 \cdot 03-0 \cdot 1$ and $<0.45 \mathrm{~km}^{2}$, respectively. This similarity in development conditions leads to similarities in $T_{\mathrm{c}}$ estimations.

Conversely, the Espey, Flavell, Haktanir-Sezen, Sheridan and Simas-Hawkins equations, due to having the lowest overall rankings, did not result in reasonable estimations. The considerable biases in these equations are certainly the result of one or more reasons. 
Table 5. Rank and correction factors of the selected equations

\begin{tabular}{|c|c|c|c|c|c|c|c|}
\hline \multirow[b]{2}{*}{ Equation } & \multicolumn{6}{|c|}{ Goodness-of-fit indices } & \multirow[b]{2}{*}{ Sum of ranks } \\
\hline & RMSE: $h$ & Rank & ME or bias: $\min$ & Rank & MRE or SBS: $\%$ & Rank & \\
\hline Arizona DoT & 0.58 & 11 & $-31 \cdot 3$ & 11 & $-63 \cdot 4$ & 11 & 33 \\
\hline Bransby Williams & $1 \cdot 88$ & 29 & $105 \cdot 9$ & 32 & $223 \cdot 0$ & 33 & 94 \\
\hline Basso & $0 \cdot 28$ & 7 & $16 \cdot 3$ & 8 & $35 \cdot 8$ & 9 & 24 \\
\hline California & $0 \cdot 17$ & 4 & $5 \cdot 9$ & 3 & $11 \cdot 0$ & 3 & 10 \\
\hline Carter & $0 \cdot 28$ & 8 & $-13 \cdot 9$ & 7 & $-26 \cdot 0$ & 6 & 21 \\
\hline Corps of Engineers & 0.75 & 16 & $43 \cdot 3$ & 16 & 95.6 & 18 & 50 \\
\hline Desbordes & 0.87 & 21 & $50 \cdot 0$ & 22 & $111 \cdot 3$ & 21 & 64 \\
\hline DNOS & $0 \cdot 13$ & 2 & $5 \cdot 9$ & 4 & $14 \cdot 4$ & 5 & 11 \\
\hline Dooge & 0.75 & 17 & $44 \cdot 5$ & 18 & $109 \cdot 4$ & 20 & 55 \\
\hline Eagleson & 0.22 & 6 & $-11 \cdot 8$ & 6 & $-30 \cdot 6$ & 8 & 20 \\
\hline Epsey & $22 \cdot 59$ & 36 & $132 \cdot 8$ & 36 & $295 \cdot 6$ & 36 & 108 \\
\hline Epsey-Winslow & 0.80 & 19 & $-44 \cdot 0$ & 17 & $-90 \cdot 8$ & 15 & 51 \\
\hline FAA & 0.76 & 18 & $43 \cdot 1$ & 15 & 92.6 & 16 & 49 \\
\hline Flavell & 8.03 & 34 & $79 \cdot 3$ & 25 & $113 \cdot 2$ & 23 & 82 \\
\hline Giandotti & 0.82 & 20 & $48 \cdot 5$ & 21 & $112 \cdot 8$ & 22 & 63 \\
\hline Haktanir-Sezen & $3 \cdot 54$ & 33 & $109 \cdot 8$ & 35 & $274 \cdot 1$ & 35 & 103 \\
\hline Johnstone-Cross & $1 \cdot 74$ & 28 & $100 \cdot 9$ & 31 & $221 \cdot 7$ & 32 & 91 \\
\hline Kerby-Hathaway & 0.58 & 12 & $34 \cdot 4$ & 12 & $79 \cdot 2$ & 14 & 38 \\
\hline Kirpich-Pen & 0.63 & 13 & $-35 \cdot 5$ & 13 & $-75 \cdot 2$ & 12 & 38 \\
\hline Kirpich-Ten & $0 \cdot 16$ & 3 & 6.05 & 5 & $11 \cdot 3$ & 4 & 12 \\
\hline McCuen et al. & 1.66 & 26 & $97 \cdot 3$ & 29 & $215 \cdot 6$ & 31 & 86 \\
\hline Pasini & $1 \cdot 23$ & 24 & $62 \cdot 4$ & 23 & $122 \cdot 6$ & 24 & 71 \\
\hline Pickering & $0 \cdot 17$ & 5 & $5 \cdot 8$ & 2 & $10 \cdot 9$ & 2 & 9 \\
\hline Picking & 0.07 & 1 & $2 \cdot 61$ & 1 & $6 \cdot 2$ & 1 & 3 \\
\hline Pilgrim-McDermott & $1 \cdot 17$ & 23 & $68 \cdot 6$ & 24 & $174 \cdot 8$ & 25 & 72 \\
\hline Ribeiro & 1.63 & 25 & $95 \cdot 3$ & 27 & $211 \cdot 7$ & 30 & 82 \\
\hline Schaake et al. & 0.41 & 9 & $-19 \cdot 9$ & 10 & $-36 \cdot 3$ & 10 & 29 \\
\hline SCS-lag & $3 \cdot 12$ & 32 & $98 \cdot 6$ & 30 & $187 \cdot 8$ & 26 & 88 \\
\hline SCS-ave velocity & 0.48 & 10 & $17 \cdot 7$ & 9 & $29 \cdot 4$ & 7 & 26 \\
\hline Sheridan & $14 \cdot 51$ & 35 & $85 \cdot 0$ & 26 & $188 \cdot 9$ & 27 & 88 \\
\hline Simas-Hawkins & $2 \cdot 67$ & 31 & $108 \cdot 6$ & 34 & $188 \cdot 9$ & 28 & 93 \\
\hline Temez & 1.66 & 27 & $95 \cdot 5$ & 28 & $207 \cdot 2$ & 29 & 84 \\
\hline Van Sickle & 0.72 & 15 & $-45 \cdot 7$ & 19 & -94.9 & 17 & 51 \\
\hline Chow et al. & 0.65 & 14 & $35 \cdot 9$ & 14 & $75 \cdot 7$ & 13 & 41 \\
\hline Ventura & 0.90 & 22 & $46 \cdot 2$ & 20 & $95 \cdot 6$ & 19 & 61 \\
\hline Williams & $1 \cdot 88$ & 30 & 106 & 33 & $223 \cdot 2$ & 34 & 97 \\
\hline
\end{tabular}

Firstly, dependence on only one parameter and limitations of the $T_{\mathrm{c}}$ equations reduce the applications of $T_{\mathrm{c}}$ equations to other catchments. For example, the Flavell and HaktanirSezen equations only depend on the river length whereas the Sheridan formula is only based on catchment area. In addition, in all the $T_{\mathrm{c}}$ equations that contain the river length parameter, the $T_{\mathrm{c}}$ value increases as river length increases and vice versa but, in the Simas-Hawkins equation, the relation between $T_{\mathrm{c}}$ and river length parameter is reversed.

Secondly, the $T_{\mathrm{c}}$ equations may have been calibrated for a limited range of input parameters that differ substantially from the values of the study area. For instance, the Sheridan and Haktanir-Sezen' formulas were developed for large and very large catchments, while the size of the Meime watershed is relatively small.

Thirdly, the empirical coefficients (most of them are land cover-related coefficients) may reflect regional effects, which make them inappropriate to use in other regions. For example, the Pasini' formula was based on data from specific watersheds in Italy and the Ribeiro' equation was developed based on data obtained from eight rural basins in the USA and India. Therefore, the empirical coefficients (constant or exponent coefficients) in these formulas may be unusable for regions similar to the MRB.

Any of these reasons may lead to significant biases in the $T_{\mathrm{c}}$ equations and the low performances are an indication that the $T_{\mathrm{c}}$ formulas are sensitive and should be evaluated or corrected before being used for other locations.

\subsection{Classification of the $T_{\mathrm{c}}$ equations}

All the $T_{\mathrm{c}}$ equations were classified based on Euclidean distance and the Ward method. Using Equation 5, the RMSSTD index was calculated for all possible numbers clusters. As shown in Figure 3, the RMSSTD decreased with the number of clusters and remained almost constant after a specific 


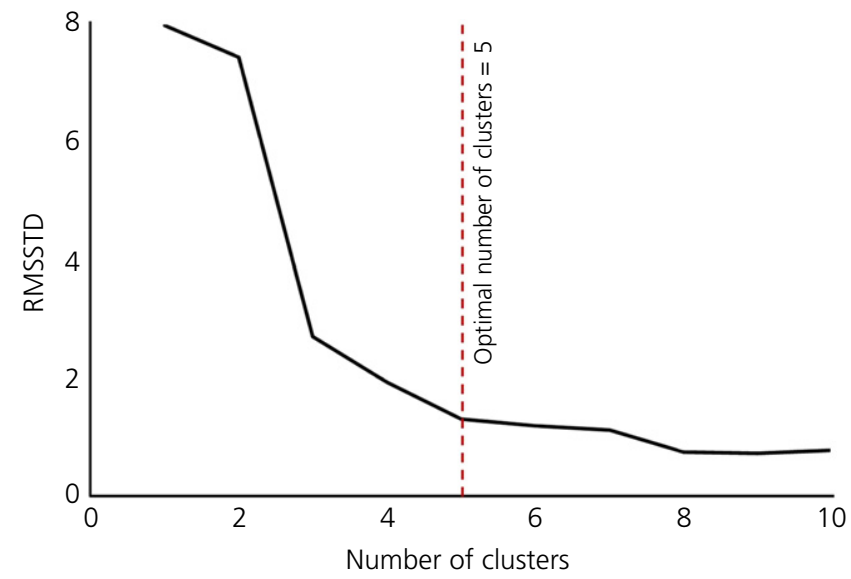

Figure 3. Variation of RMSSTD with number of clusters

number, which indicates the optimal number of clusters for classifications. In this study, five clusters were accepted for classification of the $T_{\mathrm{c}}$ equations. Figure 4 shows the clustering diagram generated based on hierarchical CA with an added line indicating the optimal stopping point of the clustering procedure.

Based on the most similarity, the following five clusters were obtained.
Cluster 1. This cluster includes nine equations with the highest similarity. One of the most important factors for this similarity is related to the fact that all of these equations were developed for rural or very small catchments. For instance, the SCS-lag/Ribeiro (USA) and Ventura/Pasini (Italy) formulas were developed for rural catchments. In other words, the conditions for the development of the equations in cluster 1' were very similar to each other. Moreover, investigations of the geomorphological parameter exponents of all the equations in this cluster show that there is a downward trend in the curve of flow path slope and river length exponents. It can be concluded from Figure 5(a) that, by increasing the river length exponent from $0 \cdot 22$ to $0 \cdot 78$, the slope exponent decreases from -0.06 to -0.38 .

Meanwhile, statistical analyses reveal that the minimum, maximum and average values of $T_{\mathrm{c}}$ in this cluster are about 5.04, 7.80 and 6.35 h, respectively (Table 6). The coefficient of variation $(\mathrm{CoV})$ of this cluster is less than that of the other clusters, with a value of about $14 \cdot 5 \%$.

- Cluster 2. This cluster contains seven equations with the highest similarity. Most of the equations in this cluster are based on data obtained from agricultural, rural and small urban catchments in the USA. In contrast to cluster 1, there is an upward trend between the river length and flow path slope exponents, as shown in Figure 5(b). In this

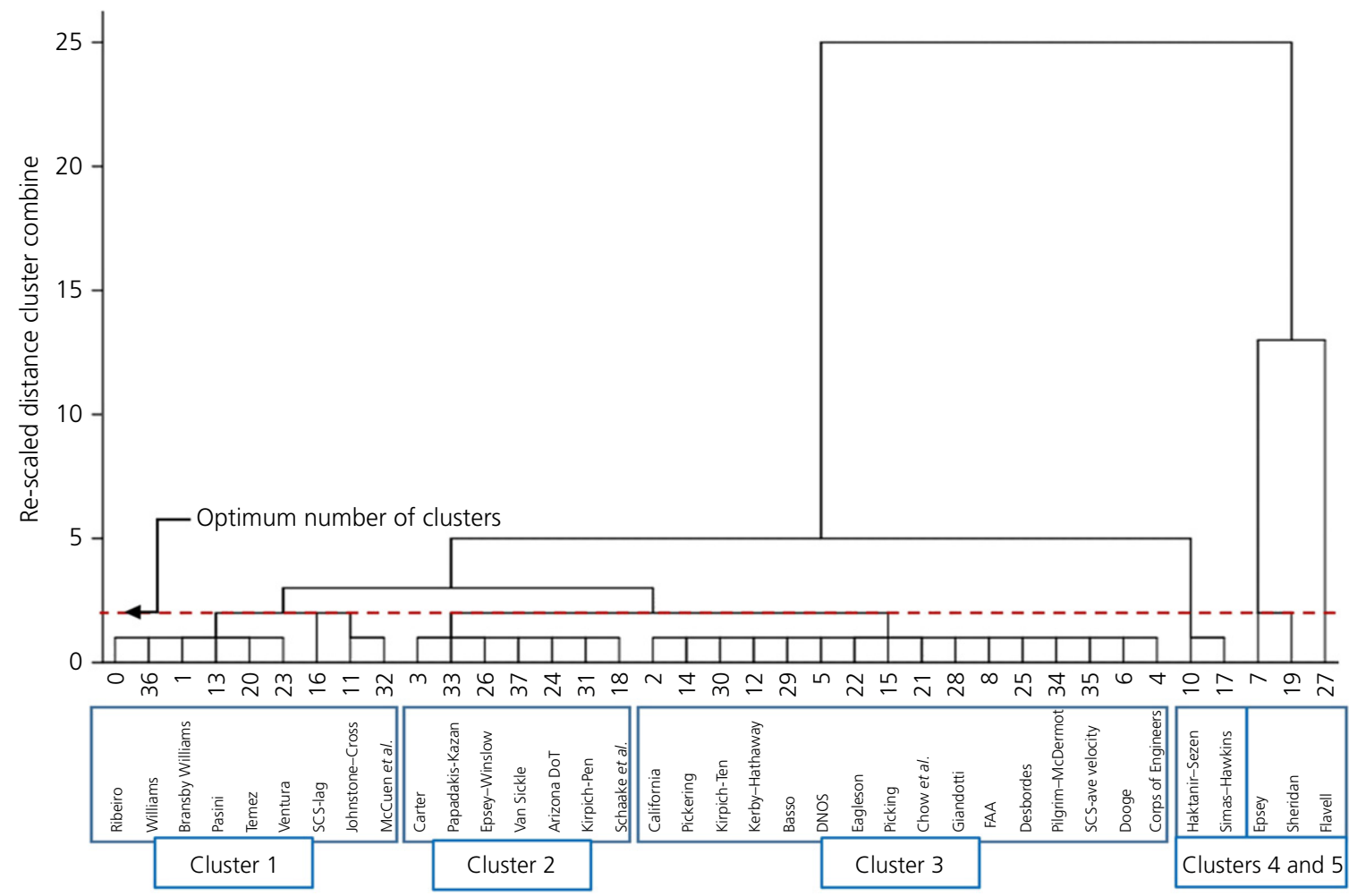

Figure 4. Clustering diagram with line added indicating optimal number of clusters 


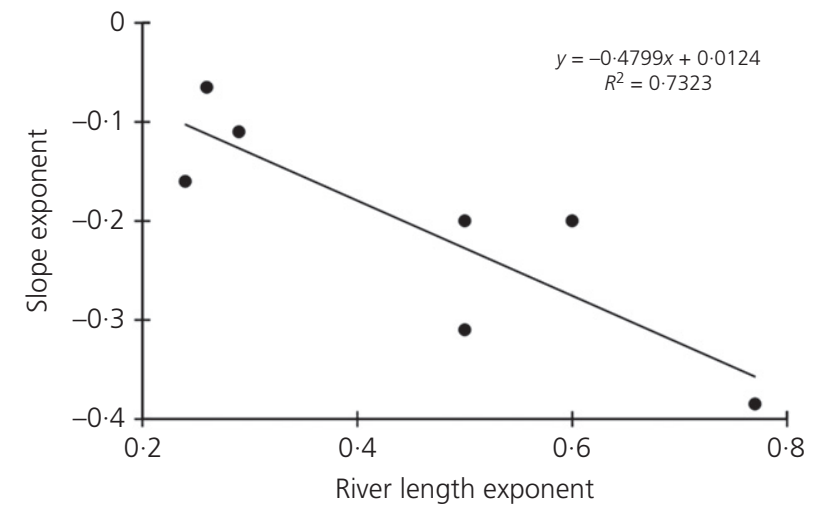

(a)

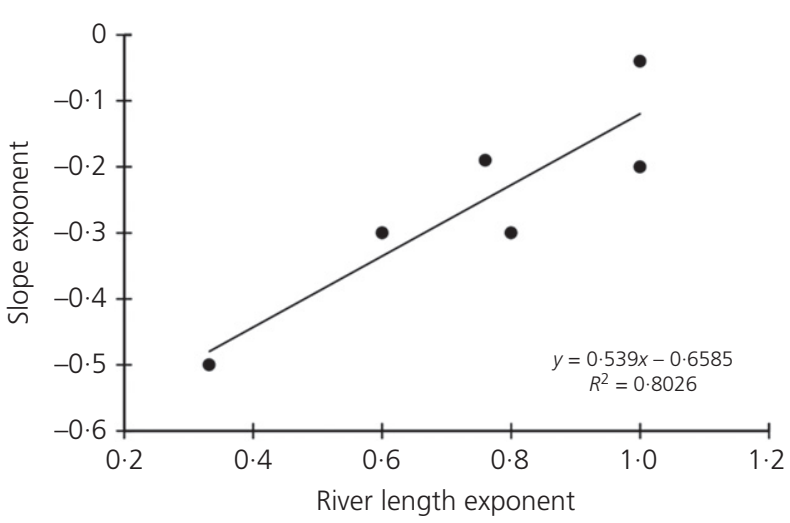

(b)

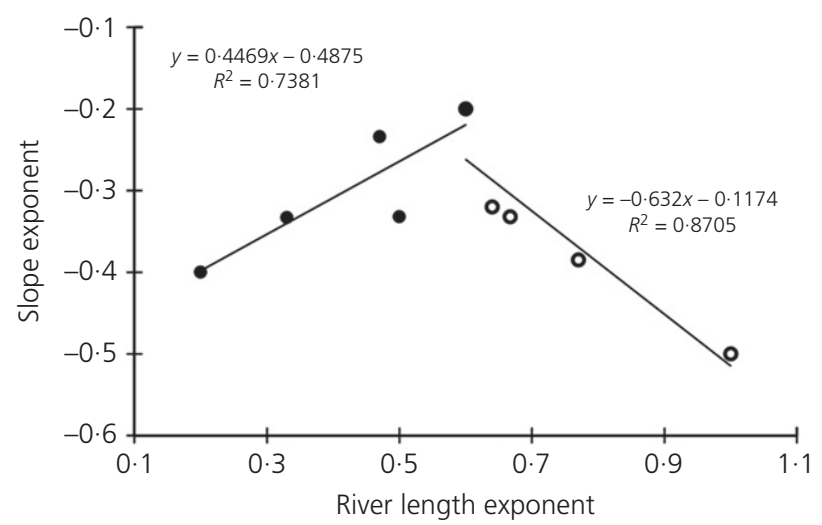

(c)

Figure 5. Relation between slope and river length exponents: (a) cluster 1; (b) cluster 2; (c) cluster 3

Table 6. Clustered $T_{\mathrm{c}}$ equations and their statistical parameters

\begin{tabular}{|c|c|c|c|c|c|c|}
\hline Cluster & Equation & Min: $h$ & Mean: $\mathbf{h}$ & Max: $\mathrm{h}$ & $\begin{array}{l}\text { Standard } \\
\text { deviation: } \mathrm{h}\end{array}$ & CoV: \% \\
\hline 1 & $\begin{array}{l}\text { Ribeiro, Williams, Bransby Williams, Pasini, Temez, Ventura, } \\
\text { SCS-lag, Johnstone-Cross, McCuen et al. }\end{array}$ & $5 \cdot 04$ & $6 \cdot 35$ & $7 \cdot 80$ & 0.92 & $14 \cdot 5$ \\
\hline 2 & $\begin{array}{l}\text { Carter, Papadakis-Kazan, Epsey-Winslow, Van Sickle, Arizona } \\
\text { DoT, Kirpich-Pen, Schaake et al. }\end{array}$ & $0 \cdot 10$ & 0.69 & $1 \cdot 43$ & $0 \cdot 50$ & $72 \cdot 3$ \\
\hline 3 & $\begin{array}{l}\text { Chow et al., Giandotti, FAA, California, Pickering, Kirpich-Ten, } \\
\text { Kerby-Hathaway, Basso, DNOS, Eagleson, Picking, } \\
\text { Desbordes, Pilgrim-McDermott, SCE-ave velocity, Dooge, } \\
\text { Corps of Engineers }\end{array}$ & $2 \cdot 14$ & $3 \cdot 12$ & $4 \cdot 18$ & 0.46 & $21 \cdot 8$ \\
\hline $4-5$ & Haktanir-Sezen, Simas-Hawkins, Epsey, Sheridan, Flavell & $9 \cdot 46$ & $24 \cdot 18$ & $38 \cdot 6$ & $14 \cdot 17$ & $58 \cdot 6$ \\
\hline
\end{tabular}

cluster, by increasing the river length exponent from 0.33 to $1 \cdot 0$, the slope exponent changes from $-0 \cdot 50$ to $-0 \cdot 03$.

The minimum, maximum and average values of $T_{\mathrm{c}}$ in this cluster are about $0 \cdot 10,1.43$ and $0.69 \mathrm{~h}$, respectively. The $\mathrm{CoV}$ of this cluster is $72.3 \%$, meaning that there is a high discrepancy between the $\mathrm{CoV}$ of this cluster and the other clusters.

- Cluster 3. This cluster is the largest, with about $41 \%$ of all the $T_{\mathrm{c}}$ equations falling in this cluster. Most of the equations in this cluster were developed for steep and small mountainous catchments. Similar to the other clusters, the trend of the geomorphological parameter exponents plays an important role in the similarity between the $T_{\mathrm{c}}$ equations in this cluster. As shown in Figure 5(c), there is no unique trend between the river length and flow path slope exponents. By increasing the length exponent up to 0.55 there is an upward trend, while from $0 \cdot 55$ to 1 the trend between river length and flow path slope exponents reverses. Statistical analyses showed that the minimum, maximum and average values 
of $T_{\mathrm{c}}$ in this cluster are about $2 \cdot 14,4 \cdot 18$ and $3 \cdot 12 \mathrm{~h}$, respectively.

- Cluster 4. This cluster is the smallest cluster, comprising only two equations, the Simas-Hawkins and Haktanir-Sezen formulas. One of the main reasons for this clustering is that, unlike all the other $T_{\mathrm{c}}$ equations, in the Simas-Hawkins formula, by increasing length variable the value of $T_{\mathrm{c}}$ decreases and vice versa. In addition, in this formula, the exponent of the length variable is larger than those of the equations in clusters $1-3$, and this issue leads to a larger estimation of $T_{\mathrm{c}}$.

- Cluster 5. This cluster is also small, containing only the Epsey, Sheridan and Flavell equations. Relative to the other clusters, there is a significant difference between the estimated and observed $T_{\mathrm{c}}$ values. Statistical analyses revealed that the minimum and maximum values of $T_{\mathrm{c}}$ in this cluster are about 25.27 and $38.62 \mathrm{~h}$, respectively. The findings reveal that using formulas that depend on only one geomorphological parameter (e.g. river length (Sheridan) or catchment area (Flavell)) is not an appropriate approach for estimating $T_{\mathrm{c}}$, especially in ungauged catchments. However, according to the $T_{\mathrm{c}}$ values of these formulas and the equations in cluster 4 , it might be reasonable to say that clusters 4 and 5 are almost the same. These clusters were thus merged to form a single cluster, as shown in Table 6.

\section{Conclusions}

The limitations of several empirical/semi-empirical time of concentration $\left(T_{\mathrm{c}}\right)$ equations lead to much confusion in selection of the best formulas for estimating $T_{\mathrm{c}}$. In this study, an efficient approach, based on salt dilution tracing, was used to estimate $T_{\mathrm{c}}$ values in the Meime catchment, Iran. In addition, 36 previously proposed $T_{\mathrm{c}}$ formulas were classified. From the data obtained on seven sub-watersheds in the Meime catchment, it is possible to conclude that the most efficient $T_{\mathrm{c}}$ formulas for this region are the Picking, Pickering, DNOS, California and Kirpich-Ten equations. $T_{\mathrm{c}}$ estimates made from different empirical or semi-empirical formulas are subject to significant error when applied to a single catchment. For all the sub-basins of the Meime catchment, with a mean $T_{\mathrm{c}}$ of $48.1 \mathrm{~min}$ and a range from $28 \cdot 2-79 \cdot 1 \mathrm{~min}$, the maximum errors for the $36 T_{\mathrm{c}}$ equations were found to vary between $2 \cdot 6$ and $132.8 \mathrm{~min}$. It is obvious that errors in estimating $T_{\mathrm{c}}$ can significantly affect hydrological designs. The significant inaccuracies in the $T_{\mathrm{c}}$ equations may be due to different contributing factors. Firstly, the input variables needed for all the $T_{\mathrm{c}}$ formulas are different and, secondly, the empirical coefficients (constant or exponent coefficients) of all the $T_{\mathrm{c}}$ formulas differ because each was calibrated for a specific region with a limited range of input parameters that differ substantially from the values of other catchments.

Categorising the most similar equations revealed that there were five clusters with the highest similarity within the members of each cluster. One of the most important factors that increases these similarities is the empirical coefficients of the $T_{\mathrm{c}}$ equations. In each cluster, the relationship between the exponents of the most important geomorphological parameters and the limitations of the $T_{\mathrm{c}}$ formulas were found to be different from those in other clusters. For instance, in some clusters, the relationship between the exponents of flow path slope and river length showed an upward trend, while a downward trend was observed in other clusters. This study also revealed that equations with the same development conditions mostly fell into a specific cluster. The findings of this study clearly show that salt experiments can be used as an appropriate approach for estimation of $T_{\mathrm{c}}$ values, especially in data-sparse catchments that are challenged with many data-related problems.

\section{REFERENCES}

ADoT (Arizona Department of Transportation) (1993) Highway Drainage Design Manual Hydrology. ADoT, Phoenix, AZ, USA.

Azizian A and Shokoohi A (2015a) Effects of data resolution and stream delineation threshold area on the results of a kinematic wave based GIUH model. Water $S A$ 41(1): 61-70.

Azizian A and Shokoohi A (2015b) Investigation of the effects of DEM creation methods on the performance of a semi distributed model: TOPMODEL. Journal of Hydrological Engineering 20(11): 1-9.

Baiamonte G and Singh V (2015) Analytical solution of kinematic wave time of concentration for overland flow under green-ampt infiltration. Journal of Hydrological Engineering 21(3): 12-29.

Bondelid TR, McCuen RH and Jackson TJ (1982) Sensitivity of SCS models to curve number variation. Water Resources Bulletin 18(1): 111-116.

Carter RW (1961) Magnitude and frequency of floods in suburban areas. USGS Professional Paper 424-B, pp. B9-B11. US Geological Survey, Washington, DC, USA.

CDH (California Division of Highways) (1960) California Culvert Practice. Department of Public Works, Division of Highways, Sacramento, CA, USA

Chow VT, Maidment DR and Mays LW (1988) Applied Hydrology. McGraw-Hill, New York, NY, USA.

Church M and Kellerhals R (1970) Stream Gauging Techniques for Remote Areas Using Portable Equipment. Inland Waters Branch, Department of Energy Mines and Resources, Ottawa, ON, Canada, Technical bulletin No. 25.

De Almeida IK, Almeida AK, Anache JAA, Steffen JL and Alves Sobrinho T (2014) Estimation on time of concentration of overland flow in watersheds: a review. Geosciences 33(4): 661-671.

De Almeida IK, Almeida AK, Steffen JL and Alves Sobrinho T (2016) Model for estimating the time of concentration in watersheds. Water Resources Management 30(12): 4083-4096.

Dhakal N, Fang X, Thompson DB and Cleveland TG (2014) Modified rational unit hydrograph method and applications. Proceedings of the Institution of Civil Engineers - Water Management 167(7): 381-393, http://dx.doi.org/10.1680/wama.13.00032.

Dingman L (2002) Physical Hydrology, 2nd edn. Prentice Hall, Upper Saddle River, NJ, USA.

Dooge JCI (1973) Linear Theory of Hydrologic Systems. US Department of Agriculture, Washington, DC, USA, Technical Bulletin 1468.

Drost JW (1989) Single-Well and Multi-Well Nuclear Tracer Techniques - A Critical Review. Unesco, Paris, France.

Eagleson PS (1962) Unit hydrograph characteristics for sewered areas. Journal of the Hydraulics Division ASCE 88(2): 43-65. 
El-Hames AS (2013) Determination of time of concentration for ungauged arid region catchments. Arabian Journal for Science and Engineering 38(7): 1833-1839.

Eslamian S and Mehrabi A (2005) Determination of experimental relations in estimation of concentration time in mountainous watershed basins. Iranian Journal of Natural Resources and Agricultural Sciences 12(5): 23-34 (in Persian).

FAA (Federal Aviation Administration) (1970) Advisory Circular on Airport Drainage. Department of Transportation, Washington, DC, USA.

Fang X, Thompson D, Cleveland T and Pradhan P (2007) Variations of time of concentration estimates using NRCS velocity method. Journal of Irrigation and Drainage Engineering 133(4): 314-322.

Fang X, Thompson DB, Cleveland TG, Pradhan P and Malla R (2008) Time of concentration estimated using watershed parameters determined by automated and manual methods. Journal of Irrigation and Drainage Engineering 134(4): 202-211.

Flavell DJ (1983) The rational method applied to small rural catchments in the south west of western Australia. Journal of Civil Engineering and Transactions 25(2): 121-127.

Giandotti M (1934) Previsione delle piene e delle magre dei corsi d'acqua. Memorie e Studi Idrografici 8(2): 47-59 (in Italian).

Grimaldi S and Petroselli A (2015) Do we still need the rational formula? An alternative empirical procedure for peak discharge estimation in small and ungauged basins. Hydrological Sciences Journal 60(1): 67-77.

Grimaldi S, Petroselli A, Tauro F and Porfiri M (2012) Time of concentration: a paradox in modern hydrology. Hydrological Sciences Journal 57(1): 217-228.

Grison F, Kobiyama M and Da Silva R (2008) Conceptual investigation of time of concentration. In From Headwaters to the Ocean (Taniguchi M, Burnett WC, Fukushima Y, Haigh M and Umezawa Y (eds)). Taylor \& Francis, London, UK, pp. 271-275.

Haan CT, Barfield BJ and Hayes JC (1994) Design Hydrology and Sedimentology for Small Catchments. Elsevier, Amsterdam, the Netherlands.

Haktanir T and Sezen N (1990) Suitability of two-parameter gamma and three-parameter beta distributions as synthetic unit hydrographs in Anatolia. Hydrological Sciences Journal 35(2): 167-184.

Hathaway GA (1945) Design of drainage facilities. Transactions of the American Society of Civil Engineers 110(6): 697-733.

Hatvani IG, Kovács J, Kovács IS, Jakusch P and Korponai J (2011) Analysis of long-term water quality changes in the Kis-Balaton water protection system with time series-, cluster analysis and Wilks' lambda distribution. Ecological Engineering 37(4): 629-635.

Hotchkiss RH and McCallum BE (1995) Peak discharge for small agricultural watersheds. Journal of Hydraulic Engineering 121(1) 36-47.

Huang J, Chan Y and Lee K (2015) Real-time flood forecasting system: case study of Hsia-Yun watershed, Taiwan. Journal of Hydrological Engineering 21(4): 31-47.

Hudson R and Fraser J (2002) Alternative Methods of Flow Rating In Small Coastal Streams. Ministry of Forests, Vancouver, BC, Canada.

Johnstone D and Cross WP (1949) Elements of Applied Hydrology. Ronald Press, New York, NY, USA.

Kerby WS (1959) Time of concentration for overland flow. Civil Engineering 26(3): 60-70.

Kirpich ZP (1940) Time of concentration of small agricultural watersheds. Civil Engineering 10(6): 362-368.

Kumar B and Nachiappan RP (2000) Estimation of alluvial aquifer parameters by a single-well dilution technique using isotopic and chemical tracers: a comparison. In Tracers and Modelling in Hydrogeology (Dassargues A (ed.)). IAHS Press, Wallingford, UK, Publication 262, pp. 53-56.
Li J, Wang Y, Xie X and Su C (2012) Hierarchical cluster analysis of arsenic and fluoride enrichments in groundwater from the Datong basin, Northern China. Journal of Geochemical Exploration 118(3): 77-89.

Liang $\mathrm{J}$ and Melching C (2012) Comparison of computed and experimentally assessed times of concentration for a v-shaped laboratory watershed. Journal of Hydrological Engineering 17(12): 1389-1396.

Linsley RK, Kohler MA, Paulhus JL, Serra MF and Aparicio FD (1977) Hidrologia Para Ingenieros. McGraw-Hill, Bogota, Colombia.

Lowrimore G and Manton KG (2016) Cluster Analysis: Overview. Wiley StatsRef: Statistics Reference Online. See http://onlinelibrary.wiley. com/doi/10.1002/9781118445112.stat06490.pub2/abstract (accessed 03/10/2017).

Manoj KC, Fang X, Yi Y et al. (2013) Improved time of concentration estimation on overland flow surfaces including low-sloped planes. Journal of Hydrological Engineering 19(3): 495-508.

Mata-Lima H, Vargas H, Carvalho J et al. (2007) Comportamento hidrológico de bacias hidrográficas: integração de métodos e aplicação a um estudo de caso. Revista Escola de Minas 60(3): 40-56 (in Portuguese).

McCuen RH (1998) Hydrologic Analysis and Design. 2nd edn. Prentice Hall, Upper Saddle River, NJ, USA.

McCuen RH (2009) Uncertainty analyses of watershed time parameters. Journal of Hydraulic Engineering 14(5): 490-498.

McCuen RH, Wong S and Rawls W (1984) Estimating urban time of concentration. Journal of Hydraulic Engineering110(7): 887-904.

Melchiorre C, Matteucci M, Azzoni A and Zanchi A (2008) Artificial neural networks and cluster analysis in landside susceptibility zonation. Geomorphology 94(3-4): 379-400.

Milligan GW and Cooper MC (1985) An examination of procedures for determining the number of clusters in a data set. Psychometrika 50(2): 159-179.

Mockus V (1961) Watershed Lag. Department of Agriculture, Soil Conservation Service, Washington, DC, USA, ES-1015.

Moore RDD (2004) Introduction to salt streamflow measurement part 2: constant-rate injection. Streamline Watershed Management Bulletin 8(1): 11-15.

Moth (Ministry of Transportation and Highways) (1998) Hydraulics Manual. Moth Engineering Branch, Victoria, BC, Canada.

NRCS (National Resource Conservation Service) (1972) National Engineering Handbook. Department of Agriculture, Washington, DC, USA.

NRCS (1986) Urban Hydrology for Small Watersheds. NRCS Washington, DC, USA, Technical Release 55.

O'Callaghan JF and Mark DM (1984) The extraction of drainage network from digital elevation data. Computer Vision, Graphics, and Image Processing 28(1): 393-344.

Ogbonna S (2004) Formula for the time of concentration of runoff. Journal of Hydraulic Engineering 130(6): 576-579.

Pasini F (1914) Relazione sul Progettodella Bonifica Renana. Bologna University, Bologna, Italy, Report (in Italian).

Pilgrim DH and McDermott GE (1982) Design floods for small rural catchments in eastern New South Wales. Institution of Civil Engineers of Australia Civil Engineering Transactions 24(4): 226-234.

Ratrout N (2010) Subtractive clustering-based k-means technique for determining optimum time-of-day breakpoints. Journal of Computing in Civil Engineering 25(5): 380-387.

Ribeiro G (1961) Acerca do calculo da vasão de obras d'arte : tempo de concentração. Revista do Clube de Engenharia 294(3): 16-19 (in Portuguese).

Sadeghi SHR, Mostafazadeh R and Sadoddin A (2015) Changeability of simulated hydrograph from a steep watershed resulted from 
applying Clark's IUH and different time-area histograms. Environmental Earth Sciences 74(4): 3629-3643.

Schaake JG, Geyer JC and Knapp JW (1967) Experimental examination of the rational method. Journal of the Hydraulics Division ASCE 93(6): 353-370.

Sharma SC (1995) Applied Multivariate Techniques. Wiley, New York, NY, USA.

Sharifi S and Hosseini SM (2011) Methodology for identifying the best equations for estimating the time of concentration of watersheds in a particular region. Journal of Irrigation and Drainage Engineering 137(11): 712-719.

Sheridan J (1994) Hydrograph time parameters for flatland watersheds. Transactions of the American Society of Agricultural Engineers 37(1): 103-113.

Silveira ALL (2005) Performance of time of concentration formulas for urban and rural basins. Brazilian Journal of Water Resources 10(5): 5-23 (in Portuguese with English abstract).

Simas MJ and Hawkins RH (2002) Lag time characteristics in small watersheds in the United States. Proceedings of the 2th Federal Interagency Hydrologic Modeling Conference, Las Vegas, NV, USA. The University of Arizona, Tucson, AZ, USA.

Singh VP (1988) Hydrologic Systems: Rainfall-Runoff Modeling, vol. 1, Prentice Hall, Englewood Cliffs, NJ, USA.
Spence C and McPhie M (1997) Streamflow measurement using salt dilution in tundra streams, northwest territories, canada1. Journal of the American Water Resources Association 33(2): 285-291.

Tazoil A (2011) Experimental methods for river discharge measurements: comparison among tracers and current meter. Hydrological Sciences Journal 56(7): 1314-1324.

Temez JR (1978) Calculo Hidrometeorologico de Caudales Maximos em Pequeñas Cuencas Naturales. Ministério de Obras Publicas y Urbanismo (MOPU), Direccion General de Carreteras, Madrid, Spain (in Spanish).

Van Sickle D (1962) The effects of urban development on storm runoff. The Texas Engineer 32(12): 23-27.

Williams GB (1922) Flood discharges and the dimensions of spillways in India. Engineering (London) 121(9): 321-322.

Wong T (2009) Evolution of kinematic wave time of concentration formulas for overland flow. Journal of Hydrological Engineering 14(7): 739-744.

Wong TSW and Asce F (2005) Assessment of time of concentration formulas for overland flow. Journal of Irrigation and Drainage Engineering 131(4): 383-387.

Yoo C, Ku H and Kim K (2011) Use of a distance measure for the comparison of unit hydrographs: application to the stream gauge network optimization. Journal of Hydrological Engineering 16(11): $880-890$.

\section{How can you contribute?}

To discuss this paper, please email up to 500 words to the editor at journals@ice.org.uk. Your contribution will be forwarded to the author(s) for a reply and, if considered appropriate by the editorial board, it will be published as discussion in a future issue of the journal.

Proceedings journals rely entirely on contributions from the civil engineering profession (and allied disciplines). Information about how to submit your paper online is available at www.icevirtuallibrary.com/page/authors, where you will also find detailed author guidelines. 